\begin{tabular}{|c|c|c|}
\hline $\begin{array}{l}\text { EXCELLENT } \\
\text { PUBLISHERS }\end{array}$ & $\begin{array}{l}\text { International Journal of Current Research } \\
\text { and Academic Review } \\
\text { ISSN: 2347-3215 (Online) } \\
\text { Journal homepage: holtp://www.ijcrar.com } 5 \text { (May-2017) }\end{array}$ & 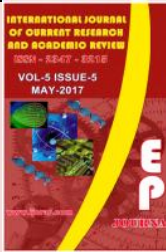 \\
\hline
\end{tabular}

doi: https://doi.org/10.20546/ijcrar.2017.505.006

\title{
A Review on Cosmic Inflation
}

\author{
Nirakar Sapkota ${ }^{1 *}$ and Binod Adhikari ${ }^{1,2}$ \\ ${ }^{I}$ Department of Physics, St. Xavier's College, Maitighar, Kathmandu, Nepal \\ ${ }^{2}$ Department of Physics, Patan Multiple College, Patandhoka, Lalitpur, Nepal
}

*Corresponding author

\section{Abstract}

The Standard Big Bang Cosmology gives the most accepted concept about the beginning and evolution of the Universe. However, it has problems: the flatness problem, the horizon problem and the monopole problem. The predictions of the Standard Big Bang Cosmology do not match the observations of modern cosmologists. Nonetheless, the admirers of the Standard Big Bang Cosmology continued to find out ways for solving those problems and such attempts lead to our knowledge of Inflationary Cosmology. The theory of inflation, which was first proposed by Alan Guth in 1981, soon became a "need" of modern cosmology and various modified models of inflationary Universe were proposed. In this paper, the author gives a brief insight of the Standard Big Bang Cosmology, introduces inflationary cosmology with its brief background, reviews some concepts associated with cosmic inflation, explains how inflation can be classified into various types, describes few of the popular types in brief and explains how the cosmological problems are solved by cosmic inflation. Moreover, few insightful examples have been given to easily explain the fundamental concepts so that even a junior researcher can get thorough idea about the field by escaping the equations and simply going through the text selectively.
\end{abstract}

\section{Article Info}

Accepted: 05 May 2017

Available Online: 20 May 2017

\section{Keywords}

Cosmic inflation, Horizon problem, Flatness problem, Monopole problem.

\section{Introduction}

The evolution of ideas across time lead to the proposal of Big Bang Theory as an approach to the logic that if the Universe is expanding as discovered by Hubble, the whole Universe must have begun from a single place in the past and expanded to become as big as it is today. However, the Big Bang model in itself wasn't complete, it had particularly three problems. The first one is called the "Horizon Problem". The Universe is homogeneous and isotropic in all directions. Even distant regions of the Universe which haven't come in contact by any means have same temperature and other physical properties when one observes the Cosmic Microwave Background.
Observation of a patch on $\mathrm{CMB}$ in one direction and another patch on CMB in exactly the opposite direction is equivalent to looking at points 27.6 billion years far (13.8 billion years far in each direction). Even light hasn't had enough time to travel from one of these points to another since the Big Bang which happened only 13.8 billion years ago. However, the temperature deviates no more than a thousandth of a degree which is a difficult thing to explain. Since the speed of light is the ultimate speed of information exchange, such an isotropy and uniformity in $\mathrm{CMB}$ was a problem for the Cosmologists to deal with. The situation gets even worse when we try to comprehend after looking at the sky 380,000 years after the big bang when CMB radiation was first formed. 
Observations of opposite points in the sky are separated by 90 million light years and yet, they are uniform. This means that within 380,000 years, light had to travel 90 million light years which isn't possible (MichioKaku, 2004). The second problem is called the "Flatness Problem". In the 1960s, it was discovered that the density of matter in the Universe was comparable to the critical density. Now, we know that $\Omega_{0}$ is 1 within $1 \%$ error which indicates a flat geometry of the Universe. This implies that in the past, the density of mass and energy must have been even closer to the critical density. i.e. $\Omega$ had to be equal to 1 within one part in a hundred trillion (MichioKaku, 2004). Such a "fine-tuning" of the Universe seems very unlikely to the scientists, especially because we generally don't like to go against the Cosmological Principle.The third problem is called the "Monopole Problem". Many GUT theories had a common prediction- the copious production of number of monopoles in the Big Bang. It is predicted that they should have been produced at high temperature and existed to this date in such quantity that they would be the primary constituent of the Universe (Preskill and John, 1979). However, even after centuries of experiments, no evidence for the existence of a magnetic monopole has been found. However, all three of these problems can be solved by an inflationary model of the Universe i.e. by assuming that from $10^{-36}$ to $10^{-33}$ or $10^{-32}$ seconds after the Big Bang, the Universe expanded by a factor of $10^{50}$. The explanation of how the "Horizon problem", the "Flatness problem" and the "Monopole Problem" are solved by an inflationary model is given in this review, along with some description of the inflationary model itself. At last, an alternative to cosmic inflation is suggested. In this paper, we discuss the features of the standard big bang cosmology in the upcoming chapter. After that, we discuss about inflationary cosmology and how it solves the problems of standard big bang cosmology. At last, we present a conclusion along with our hypothesis regarding cosmic inflation.

\section{Standard big bang cosmology}

The Universe is isotropic and homogeneous on the largest scales as suggested by the Cosmological Principle which is why the metric takes the Friedman RobertsonWalker (FRW) form:

$d s^{2}=-d t^{2}+a^{2}(t)\left[\frac{d r^{2}}{1-K r^{2}}+r^{2}\left(d \theta^{2}+\sin ^{2} \theta d \phi^{2}\right)\right]$
Where $a(t)$ is the scale factor and $t$ is the cosmic time. The constant $\mathrm{K}$ is related to the geometry of the Universe and its value can be negative (corresponding to an open hyperbolic Universe), zero (corresponding to a flat Universe) and positive (corresponding to a closed Universe). It turns out that the Universe is flat on the largest scale i.e. the $\mathrm{K}=0$ metric is suitable. Matter in the Universe determines the fate of the Universe. Following is a relation between pressure $p$ and the energy density $\rho(\mathrm{t}): p=\frac{\rho}{3}$ for Radiation and, $p=0$ ofor matter. The Universe may be made up of several types of material, radiation and non-relativistic matter which is why a simple equation of state is not necessary. Einstein's field equations should be solved for determining how Universe evolves over time.

$G_{\mu v} \equiv R_{\mu v}-\frac{1}{2} g_{\mu v} R=8 \pi G T_{\mu v}-\Lambda g_{\mu v}$

Considering that the cosmological constant is negligible, we get:

$H^{2}=\frac{8 \pi}{3 m^{2} p I} \rho-\frac{K}{a^{2}}$

$\dot{\rho}+3 H(\rho+p)=0$

Where $\mathrm{H}$ is the Hubble parameter (also known as Hubble Expansion Rate) that is equal to $\frac{a}{a}$. Equation (3) is known as Friedman Equation and Equation (4) is called Fluid Equation. The $3 \mathrm{H} \rho$ term in fluid equation is the change in density with increase in scale factor of the Universe i.e. it corresponds to a decrease in density. $3 \mathrm{Hp}$ term refers to the change in energy with respect to increase in scale factor which also corresponds to a reduction because as the space expands, the energy is utilized for doing work. From (3) and (4), we get the acceleration equation:

$\frac{\ddot{a}}{a}=-\frac{4 \pi}{3 m^{2} p I}(\rho+3 p)$

Equation (3) can be written in terms of density parameter $\Omega$ as:

$\Omega-1=\frac{K}{a^{2} H^{2}}$

Where $\Omega \equiv \frac{\rho}{\rho_{c}}, \quad \rho_{c}$ is the critical density. For a flat geometry of the Universe, $\mathrm{k}=0$ which gives the following solutions from equation (3) and (4) for matter dominated 
Universe $(\mathrm{p}=0)$ and radiation dominated Universe $(\mathrm{p}=\rho / 3)$

$\begin{array}{ll}\text { Matter Dominated Universe: } \rho \alpha \mathrm{a}^{-3} & \mathrm{a}(\mathrm{t}) \alpha \mathrm{t}^{2 / 3} \\ & \begin{array}{ll}(7) \\ \text { Radiation Dominated Universe: } \rho \alpha \mathrm{a}^{-4} & \mathrm{a}(\mathrm{t}) \alpha \mathrm{t}^{1 / 2}\end{array}\end{array}$

(8)

Solutions for the cosmological constant for which $\omega=-1$ (i.e. the ratio of pressure to energy density is -1 ) is given as:

$a(t) \alpha \exp (\mathrm{Ht})$

\section{Brief history of the universe}

The present Universe is dominated by non-relativistic matter since the density decreases more quickly with increase in scale factor as indicated by equation (8). However, in the early times, radiation dominated the Universe. The following relation explains the relationship between time and temperature in that era:

$\frac{t}{1 \sec } \approx\left(\frac{10^{10} K}{T}\right)^{2}$

Brief history of the events after the Big Bang (upto three minutes) is given below (MichioKaku, 2004):

\section{Before $\mathbf{1 0}^{-43}$ seconds-Planck era}

We are sure about almost nothing in this era. The fundamental forces of the Universe were unified (as superforce) and the gravitational force was as strong as other forces. There existed a special type of symmetry known as "Super-symmetry" and this symmetry broke down forming a bubble with the size of plank length. Plank energy $=10^{19} \mathrm{GeV}$

\section{$10^{-43}$ seconds-GUT (Grand Unified Theory) era}

In this era, symmetry breaking occurred which separated gravity from other three fundamental forces. However, Electromagnetism, strong nuclear force and weak nuclear force were still bound by GUT symmetry. Universe underwent inflation that increased its size by a factor of $10^{50}$. Temperature $=10^{32}$ degrees.

\section{$10^{-34}$ seconds - end of inflation}

The strong force split off the other two forces and the temperature dropped to $10^{27}$ degrees. Inflation ended allowing the Universe to expand normally. The Universe was about the size of the current solar system and it consisted hot plasma of free gluons, quarks (which condensed into neutrons and protons of today) and leptons. Matter and anti-matter annihilation took place, leaving only a tiny amount of matter that exceeded their anti-matter counterparts. Hopefully, this energy will be duplicated in the LHC in a couple of years.

\section{Three minutes- nuclei form}

The temperature was low enough to allow the neutron and proton to bind together for forming deuterium atom. The deuterium then combined to form He nuclei and little amount of $\mathrm{Li}$ was also formed but the further fusion process was obstructed as nuclei with 5 particles (neutrons protons) were unstable. The Universe was opaque as the radiation was scattered off by the electrons (since the atoms were in ionized state due to high temperature)

\section{Problems with the Big Bang}

The standard big bang cosmological model explains many phenomena related to the beginning of the Universe reasonably well and in experimentally verifiable manner, especially the events after few seconds of the big bang. This is why the Big Bang theory is very widely popular and the most accepted model regarding the beginning of the Universe. However, it has three major problems which were considered to be the drawbacks of this model, until the inflationary cosmology solved these problems. As a result, the Standard Big Bang Cosmology got even stronger in its foundation such that any successful theory to explain the origin of the Universe must solve these three problems. In other words, these three problems proved to be the strength of Big Bang Cosmology after the discovery of inflationary cosmology. Those three major problems of the Big Bang Cosmology are:

\section{The flatness problem}

Without taking into consideration the effects of cosmological constant, the Friedmann Equation becomes:

$\left[\Omega-1 \mid=\frac{\|k\|}{a^{2} H^{2}}\right.$

The term $a^{2} H^{2}$ in the standard big-bang evolution keeps on decreasing. This increases the value of $\Omega$ and so, the 
value of $\Omega$ keeps on shifting away from one. However, we observe that $\Omega$ is within an order of magnitude of one- which is strange because this implies that its value had to be even closer in the past. At the epoch of nucleosynthesis, $|\Omega-1|<O\left(10^{-16}\right)$ is the required condition (Liddle, 1996) and at the Plank epoch, $|\Omega-1|<O\left(10^{-64}\right)$ is the required condition (Riotto, 2002). This clearly indicates that a very fine-tuning of the conditions in the early Universe is required for the current value of $\Omega$ to match our observations. Otherwise, the Universe would soon collapse after the Big Bang or expand quickly- not allowing any structures to form.

For simplicity, it can be compared to a handkerchief half by two people such that it is stretched. If more handkerchiefs are created within, a lag should be produced. Similarly, the expansion of the Universe can be thought to create such lag in the flatness of the Universe- thus making it less flat. However, the Universe still being precisely flat is similar to a handkerchief whose when four ends are fixed and more handkerchief is being created in the middle and yet, it doesn't lag. This is weird, which is why we call it "Flatness Problem".

\section{The horizon problem}

Physical wavelength a $\lambda$ be considered which grows over time as $\mathrm{a} \lambda \alpha \mathrm{t}^{\mathrm{p}}$ (where $0<\mathrm{p}<1$ ) but Hubble Radius evolves as $\mathrm{H}^{-1} \alpha$ t. Clearly, evolution over time results in physical wavelength becoming lesser than the Hubble radius. So, places where causality works ultimately become small fraction of $\mathrm{H}^{-1}$.

Particle Horizon $D_{H}(t)$ where photons travel from the Universe's beginning, $\mathrm{t}=\mathrm{t}_{*}$

$\mathrm{D}_{\mathrm{H}}(\mathrm{t})=\mathrm{a}(\mathrm{t}) \mathrm{d}_{\mathrm{H}}(\mathrm{t}), \quad$ where $\mathrm{d}_{H}(\mathrm{t})=\int_{t_{*}}^{t} \frac{d t}{a(t)}$

$d_{H}(t)$ is the commoving distance. If $t_{*}=0, d_{H}(t)=3 t$ for an era dominated by matter. Photons in the CMB are emitted in the epoch of recombination when decoupling occurred. The ratio of commoving distance at the time of decoupling to that at present is estimated to be about $10^{-2}$ which means the causality region of photons small. Clearly, any region which are more than 2 degrees apart in CMB are causally separated at decoupling. However, the Universe is homogeneous as the CMB photons across all the sky are in thermal equilibrium with one another. This is known as "The Horizon Problem" of the Standard Big Bang Cosmology.
For simplicity, example of two walls can be taken. If a ball is thrown from wall $\mathrm{A}$ and the next wall $\mathrm{B}$ which is close to A moves away at speed greater than that of the ball, the ball won't ever strike the wall B. Similarly, the expansion of the Universe doesn't allow the thermalization of two distant points on CMB. However, accurate thermalization indicates that the ball has in fact struck the wall B!

\section{The monopole problem}

One of the many "unwanted relics" predicted by many modern particle theories is the existence of magnetic monopoles, while violates the current observations provided that scientists haven't yet discovered any magnetic monopole. Theories predict that these monopoles were created in the early age of the Universe at the time of breaking of super symmetry. Since these monopoles (which behave as matter component) are diluted slower than radiation, we expect them to dominate the present Universe. This completely contrasts with our observations. This problem in Standard Big Bang Cosmology is called "The Monopole Problem".

Example of a table in a conference room can be taken. Let, the four ends have four apples which be considered as magnetic monopoles. The room be considered as our horizon (since we can't see beyond the room). If the walls of the room expand along with the table (but not the apple i.e. apple doesn't expand in this consideration), the table's edges will never cross the room. This means the monopoles should be within our horizon. Yet, we don't see them. This is the Monopole Problem.

\section{Inflationary cosmology}

Cosmological phase transition leads to the change in the energy density of scalar field which makes it behave like cosmological constant (Linde, 1974). From false vacuum, the first order transition of phase takes place which is the cause of discontinuous changes included in some cases. An exponentially expanding theoretical Universe having false vacuum is too inhomogeneous once the bubble walls collide, which was a drawback Gennady Chibisov and Linde (1978) faced on their attempt to build such model of the Universe. This discouraged them to pursue their own idea (Linde, 2008). However, Alexei Starobinsky introduced a somewhat realistic yet complicated model incompativly with the objectives of inflation in 1979-80 (Starobinsky, 1979, 1980). The Universe was already considered isotropic 
and homogeneous since its start which was not only an ineffective way that escaped from explaining the horizon problem but also an inappropriate way which contrasts with the objective of inflationary theories. Alan Guth in 1981 highlighted a simple inflationary model that involved supercooling at the time of cosmological phase transition. (Guth, 1981) The exponential expansion of the Universe takes place in a super-cooled metastable state of false vacuum as per his theory and such Universe involves no particles as well as field but only enormous energy density. The energy density doesn't vary as the empty space doesn't get filled during expansion and this is actually the cause of exponential expansion. A rapidly blown balloon appears to have flattened suddenly for an ant on its surface and in a similar way, the rapidly expanding Universe became flat in its geometry as we observe now. Finally, the decay of false vacuum, collision of new phase bubbles and the increase in temperature of Universe take place. Guth's model is currently known as "old inflation" and is problematic when we consider the probability of formation of bubble to be high or less. If we consider that formation of bubble involves high probability, inflation should be very short as new phase bubbles form near each other. This leads to the problems of Standard Cosmology not being solved and the Universe being anisotropic as well as inhomogeneous. In addition, low probability for bubble formation implies that they are created far from each other. This makes inflation long and a Universe having very small $\Omega$ is represented by each bubble. This means that this model can neither be improved nor does it work which is why we say it faces the graceful exit problem (Linde, 2008). Only in 1981-82 was the problem fixed as the New theory of inflation described that inflation begins only in presence of unstable state at peak of effective potential or in false vauum. Inflation field rolls down slowly in effective potential which is the cause for homogeneous Universe (Linde, 2008). Despite the popularity of this model, it has couple of drawbacks. The inflation field $(\varphi)$ is required to have a flat plateau of its effective potential when $\varphi=0$ and this is another "fine-tuning" problem scientists aren't comfortable with. This scenario is the base for many subsequent versions that describe an inflation field which isn't in thermal equilibrium with other fields and which doesn't attain such equilibrium at all. Thus, it isn't appropriate to use cosmological phase transition in this case. The problem with the old and new inflation was that they both required a Universe that was relatively homogeneous, in thermal equilibrium from the very beginning and big enough to last until the start of inflation. Clearly, assumptions favored inflation to be a mere intermediate stage of the Universe's evolution. However, a radical change was brought in 1983 by the introduction of the chaotic inflation scenario which solved the problems of the old and new inflation theory. Chaotic inflation can occur in theories with flat enough region in potential that allows slow-roll regime to exist (Linde, 1983).

\section{False vacuum}

Breaking off of a force from other forces can be compared to breaking of a dam. River flows from higher energy level (mountains) to lower energy level (sea level). The lowest energy level that is available is a true vacuum. However, a false vacuum is that state which appears to be stable but is in tremendous pressure and can break off at any time. It can be compared to the dam of a river which appears to stabilize the river but in reality, it is unstable. A small crack can burst it and cause disastrous flood leading to a transition to true vacuum. Similarly, in Grand Unified Theory, the Universe started out in a state of unstable false vacuum when the three fundamental forces of nature (except gravity) were unified as a single force. However, it was unstable like the dam and it spontaneously broke like the bursting of the dam, making transition from false vacuum where the forces were unified (like the water which was accumulated by the dam) to true vacuum where the forces are broken (the same way water got flooded).

\section{Creation of the Universe from nothing}

Even though all the matter in the Universe including rocks, planets, stars, galaxies, quasars, black holes and clusters is huge and positive, the energy stored within gravity may be negative which means the sum could add nearly to zero. This means that creation of such Universe from nothing doesn't violate the Conservation of Energy. The very idea of creating Universe from nothing was introduced by Edward Tryon who believed that the Universe "happens from time to time" as a result of quantum fluctuations in vacuum (MichioKaku, 2004). The Universe-from-nothing theory is significant to answer practical questions about why the Universe doesn't spin and why the total spin of all the galaxies in the Universe cancels out.

\section{Classification of inflation}

There are three independent ways to classify models of inflation (Linde, 1994). The first one is based upon the initial conditions to start inflation. The old and new 
inflation models assumed the Universe to be in a state of thermal equilibrium from its very beginning at an extremely high temperature. They were based upon the idea that inflation field $(\varphi)$ was in a state corresponding to minimum effective potential $\mathrm{V}(\varphi)$. Another type of inflation scenario was chaotic inflation which considered all possible initial conditions in the Universe and didn't necessary require the Universe inside the state of thermal equilibrium. This scenario didn't require the inflation field to necessarily correspond to the minimum of $V(\varphi)$ (Linde, 1983). So, this scenario includes even greater variety of models than its new inflationary model counterpart. Since the idea of thermalized beginning is nearly abandoned, all realistic models of inflation based upon first type of classification are chaotic type (Linde, 1990).

Another classification is based on various regimes that are possible during inflation: power law inflation, quasiexponential inflation, etc. This classification isn't related to initial conditions which are why there is no sense to compare power law inflation and chaotic inflation, for instance. Similarly, there is no logic in comparing new inflation with quasi-exponential inflation. These are completely different and independent modes of classification. For the same reason, inflation model with initial conditions corresponding to chaotic inflation and the regime similar to power law inflation are described as chaotic power law inflation (Linde, 1994).

The third classification is based upon how inflation ends. In literature, two possibilities are found to be discussed: slow rolling of inflation field $(\varphi)$; and first-order phase transition. The first class models include slow rolling of $\varphi$ that eventually becomes faster and faster whereas the second class should have at least two scalar fields $\varphi$ and $\sigma$. These models describe strong first-order phase transition involving production of bubble triggered by slow rolling of field $\varphi$. An example such model is 'the extended inflation scenario' that can be described as a combination of Brans-Dicke theory and the old inflationary scenario (Linde, 1994).

\section{Chaotic inflation}

Scalar field $\varphi$ with mass $m$ and potential energy density $\mathrm{V}(\varphi)$ which has a minimum value at $\varphi=0$ as

$$
V(\phi)=\frac{m^{2}}{2} \phi^{2}
$$

If the Universe doesn't expand, we expect $\varphi$ to oscillate near this minimum. In this scenario, equation of motion for $\phi$ becomes analogous to that of harmonic motion's equation, $\ddot{\phi}=-m 2 \phi$ Nonetheless, the expansion of the Universe has to be considered which is why the above equation becomes,$\ddot{\phi}+3 H \ddot{\phi}=-m 2 \phi$, where $H$ is the Hubble Constant. The term containing Hubble constant can actually be thought as some kind of friction-causing term. If the Universe is assumed to be homogeneous, then the Einstein equation corresponds as

$H^{2}+\frac{k}{a^{2}}=\frac{1}{6}\left(\dot{\phi}^{2}+m^{2} \phi^{2}\right)$

$\mathrm{M}_{\mathrm{p}}^{-2}=1$ is the units we work in.If $\varphi$ was initially large, $\mathrm{H}$ would also be large according to (13). So, the term $3 H \phi$ was also very large which means the scalar field $\varphi$ moved very slowly. This can be compared with the motion of a small ball in a viscous liquid like glycerol. The energy density of $\varphi$ remained nearly constant, leading to an expansion with much higher speed than predicted by the old cosmological theory. Such rapid increase of the scale factor of the Universe and slow motion of inflation field leads to following conditions:

$$
\underset{\phi}{\dot{\phi} 3 H \dot{\phi},} \quad H^{2} \gg \frac{k}{a^{2}},
$$

The equations can now be written as:

$H=\frac{\tilde{a}}{a}=\frac{m \phi}{\sqrt{6}}, \dot{\phi}=-m \sqrt{\frac{2}{3}}$

Clearly, slow change in the inflation field results in growth of the size of Universe as $\mathrm{e}^{\mathrm{Ht}}$. This is the stage of inflation and it ends after $\varphi$ decreases to be much less than a certain value $\left(\mathrm{M}_{\mathrm{p}}=1\right)$. Solving the above equations, we find that long stage of inflation results in exponential growth of the Universe if it is filled with scalar field $\varphi>>1$.

$a=a_{0} e^{\phi^{2 / / 4}}$

Even though there was a long debate on whether new inflation or chaotic inflation is the appropriate one, it has become clear now that chaotic inflation has much more general initial conditions and thus can easily be consistent with cosmological theories without insignificant considerations of thermal equilibrium and high temperature phase transition during the early universe. 
Chaotic inflation can describe inflation even in models with potential having flat maximum (which was originally used in new inflation). Such scenario is called "new inflation", not considering the original new inflation scenario, but according to the chaotic inflation scenario. To prevent misunderstanding of terminologies, some scientists call this version of chaotic inflation 'hilltop inflation'.

\section{The initial conditions of chaotic inflation}

We consider a closed universe having size 1 1 (in plank units) on its initial state, which comes into existence from 'nothing' in a state having Plank density $\rho \sim 1$. The sum of potential energy density, kinetic energy density and gradient energy density is given by

$\frac{1}{2} \dot{\phi}^{2}+\frac{1}{2}(\partial \mathrm{i} \phi)^{2}+V(\phi) \sim 1$

A theory with $\mathrm{V}(\varphi)=$ constant is invariant under shift symmetry $\phi \rightarrow \phi+c$ and there exists equal probability for all initial values of homogeneous component of scalar field $\phi$. A constraint appears on the field's amplitude if $\mathrm{V}(\varphi)$ is not constant and grows to become greater than the Plank density $\mathrm{V}\left(\varphi_{\mathrm{p}}\right)=1$. This implies that $\varphi$ is less than or equal to $\varphi_{p}$ but we don't have any reason to assume that $\varphi$ has to be less than $\varphi_{\mathrm{p}}$. This suggests that $\phi \sim \phi_{p}$. Initial conditions are expected to correspond to $\frac{1}{2} \phi^{2} \sim \frac{1}{2}(\partial \mathrm{i} \phi)^{2} \sim V(\phi)=O(1)$. The inflation starts when $\frac{1}{2} \phi^{2}+\frac{1}{2}(\partial i \phi)^{2} \leq V(\phi)$ and by the Plank time, the terms $\frac{1}{2} \phi^{2}$ and $\frac{1}{2}(\partial i \phi)^{2}$ decrease to values much less than $V(\phi)$ which is why inflation continues. Clearly, chaotic inflation occurs under natural initial conditions, if it can begin at $V(\phi) \sim 1$ (Linde, 2008). A small Universe can be created from nothing by the help of quantum fluctuations, given that it is done quickly so as to agree with Uncertainty relation $\Delta E \cdot \Delta \mathrm{t} \leq 1$. For a closed inflationary Universe, total energy of scalar field is proportional to the minimum volume multiplied by energy density which means such Universe can quantum mechanically come into existence within the time $\Delta t \geq 1$, assuming that $\mathrm{V}(\varphi)$ is not too small compared to Plank density.

\section{Probability of quantum creation of a Universe (closed) is proportional to}

$$
P \sim \exp \left(-\frac{24 \pi^{2}}{V}\right)
$$

Equation (17) indicates that the Universe can be formed if $\mathrm{V}$ is not too small compared to Plank density. Continuation of the Euclidean de Sitter solution to real time is possible for quantum creation of the Universe if

$\dot{\phi}=0$.

Gibbons and Turok made a remarkable attempt to evaluate the initial conditions for inflation by imposing 'initial conditions' at the end of inflation. This can be analyzed to determine the initial conditions as direction of time can be reversed to relate the end of inflation to the initial conditions. Assumption that certain conditions have equal probability of occurring at the end of inflation result in conclusion that the initial conditions suitable for inflation must have very less probability (Gibbons and Turok, 2008) However, there is a problem with this approach. A well-organized has its entropy increase, however, playing back the same scenario in reverse time will result in decrease in entropy which is misleading. Similarly, the conclusions obtained from this approach can be misleading. Moreover, the results obtained from this approach contradict the one with Euclidean approach because initial conditions of the Universe are based upon the quantum epoch near the singularity and reversing the end conditions to obtain initial conditions like the above mentioned method can be very misleading.

\section{Hybrid inflation}

Hybrid Inflation is the type of inflation which involves more than one scalar field. Most inflation models end by slow rolling mechanism of the inflation field which eventually becomes faster, or by first-order phase transition. However, one of the models of Hybrid inflation has a different end- its ends due to a very rapid rolling of scalar field $\sigma$ triggered by another scalar field $\varphi$. It is given the name "hybrid inflation" as it seems to be a hybrid of chaotic inflation with $V(\phi)=\frac{m^{2}}{2} \phi^{2}$ and usual theory with spontaneous symmetry breaking having $V(\sigma)=\frac{1}{4 \lambda}\left(M^{2}-\lambda \sigma^{2}\right)^{2}$.

The end stage of this type of inflation is not supported by the inflation potential $V(\phi)$ but by 'non-inflationary potential' $V(\sigma)$. There is another Hybrid Inflation model which involves ideas from extended inflation, new inflation and chaotic inflation. In this model, inflation ends by slow rolling which avoids big-bubble problem of extended inflation (Linde, 1994) 


\section{Eternal inflation}

Inflationary cosmology has a unique feature that involves self-reproduction of inflationary Universe which was considered to be a big problem in the old inflationary theory. Later, a similar process was introduced in the new inflationary theory. An important feature of this scenario is that Universe was divided into many exponentially large parts during the process, such that each of those parts had different properties. This justified scientifically the cosmological anthropic principle for the very first time. Anthropic principle is a philosophical approach to consider that observations in the Universe must be compatible with the life that observes it. This was initially formed as many observations showed that laws of nature and conditions of the Universe seem to take only those values that can be consistent with the parameters essential for existence of life. The probability for the existence of combination of eternal inflation and anthropic principle was first realized at the Nuffield Symposium on inflationary cosmology (Linde, 2014).

Only when the regime of self-reproduction of inflationary Universe was discovered in chaotic inflation scenario, the importance of such phenomenon was realized and it was called "eternal inflation". During the process of inflation, quantum fluctuations are produced that are responsible for increasing energy density. This leads to higher density of energy in some parts compared to the other parts of the Universe. These parts tend to expand much rapidly compared to their parent domain. Furthermore, such expansion causes even more quantum fluctuations in such parts that lead to increased energy density in some domains compared to others. In this way, there is a never-ending process which ultimately leads to division of the Universe into many parts that are exponentially large and have different laws governing the low energy state. In this scenario, the Universe becomes an inflationary multiverse (Linde, 1986, 2014).

Hubble radius $\left(\mathrm{H}^{-1}\right)$ can be defined as the distance beyond which the events are completely independent of each other. During inflationary expansion, events which are farther than the Hubble radius are separated from each other by speed faster than that of light which means information exchange is impossible between these events. Clearly, this implies that inflation in any domain is completely independent to other processes outside the Hubble radius. It is due to this reason that regions outside the Hubble radius can be considered to be a part of separate mini-Universe. This implies that the Universe would not disappear entirely but some places within the
Universe could collapse- vanishing the life on that part. However, there will always be some other places in the Universe where life will appear in different forms time and again (Linde, 2014).

Whether eternal inflation takes places only in future or it takes place even in the past is a debatable topic. Consider a time-like geodesic at the time of inflation. For observer following the geodesic, time duration $\left(t_{i}\right)$ exists which is finite. For eternal inflation to hold true, all the geodesic under consideration and the calculated $t_{i}$ should not have an upper limit. This indicates that for every time $\mathrm{T}$, geodesics with inflation for $t_{i}>T$ will exist. Few long geodesics are enough to make the space surrounding them expand exponentially which leads to eternal increase in the total volume of inflationary parts exponentially. Assumptions of geodesics in the past time in similar way can lead to proofs that the length of the geodesics is finite and yet, there is no logic to define upper bound for all the geodesics in the past (Linde, 2014). So, every part in the Universe had a beginning and will have an end for inflation but the whole Universe will not end.

In figure 2, the height in distribution represents the value of field $\varphi$ that drives inflation. The different colored paints (green, red, blue) represent three different minima of potential of field $\phi$ which determines the type of spontaneous symmetry breaking. Laws of Physics governing the low energy level are different in the context of regions represented by different colors.. The peaks in the figure (which look like mountain peaks) correspond to regions in which quantum fluctuations bring both the scalar fields back to Planck density. Each of such regions can be thought of as the beginning of a new Big Bang. The laws of physics change rapidly in those regions as shown by the changing colors but they become fixed in those parts of the Universe where $\varphi$ becomes small enough. These parts correspond to valley in the above figure. Each part grows to become exponentially large at the end of inflation, making the Universe a multiverse consisting different exponentially large local homogeneous parts having different laws of low-energy Physics.

\section{Solution to the cosmological problems}

From equation (15), the total inflation obtained starting from $V(\phi) \sim 1$ is of order $10^{10^{10}}$, as the value of mass $m$ of the scalar field is nearly $3 \times 10^{-6}$ in Plank unit. We obtain $10^{-30}$ seconds as the time period of inflation and at 
the end, the scalar field oscillates near the minimum value of potential energy density. There is loss of energy during the oscillation due to creation of pairs of elementary particles which interact with each other and attain thermal equilibrium at a temperature (say $\mathrm{T}_{\mathrm{T}}$ ) after which the Big-Bang Theory can effectively describe the evolution of the Universe (Linde, 2008).

After $10^{-30}$ seconds of inflation, even a Universe initially of Plank size $l_{p} \sim 10^{-33} \mathrm{~cm}$ attains a large size of $l \sim 10^{10^{10}} \mathrm{~cm}$. Clearly, inflation increases the size of the Universe by many orders of magnitude which is a major concept behind how inflation solves problems of the BigBang Cosmology.

Consider that our Universe initially had many domains with chaotically distributed scalar field. Then, domains with very small scalar field never inflated. This means that most of the volume of the Universe is the result of inflation of those domains which had originally large scalar field which creates huge homogeneous islands. In this context, each of the homogeneous domain becomes much larger than the observable Universe.

\section{The flatness problem}

In Equation (11), if $\mathrm{a}^{2} \mathrm{H}^{2}$ is increased, the value of $|\Omega-1|$ decreases which means $\Omega$ starts to approach unity. Since the increase in $\mathrm{a}^{2} \mathrm{H}^{2}$ is rapid, the value of $\Omega$ rapidly approaches unity. After the end of inflation, the value of $[\Omega-1 \mid$ begins to increase but the value of $\Omega$ remains closer to unity even after billions of years despite the repulsion from one for all post-inflationary period-given that the inflation is sufficient enough.

Compared to a big inflated balloon, sudden expansion can cause a surface to appear flat. This explains the flatness of the Universe.

\section{The horizon problem}

We know, scale factor a $\alpha \mathrm{t}^{\mathrm{p}}$. During inflationary epoch, $\mathrm{p}>1$. Since the Hubble radius $\mathrm{H}^{-1} \alpha \mathrm{t}$, so the physical wavelength given by a $\lambda$ increases faster than the Hubble radius. This means that during inflation, a $\lambda$ is pushed beyond the Hubble radius. This indicates that the causal region is stretched on scales much larger than $\mathrm{H}^{-1}$.

Following condition is to be satisfied for comoving particle horizon in order to solve the Horizon Problem: $\int_{t_{*}}^{t_{d e c}} \frac{d t}{a(t)} \gg \int_{t_{d e c}}^{t_{0}} \frac{d t}{a(t)}$

This means photons should travel much further before decoupling than that after decoupling. Another way to view this is based on the fact that inflation decreases comoving Hubble length which is generally a good measure of how far things can move in the Universe. Clearly, this indicates that we see much smaller region of the Universe after inflation than before inflation started. So, large smooth thermalized region were produced that encompassed a volume much larger than our present observable Universe.

As far as the example of the two walls in considered, inflationary cosmology requires the two walls to be so close to each other that the ball initially hits the second wall and later, the second wall expands exponentially (not due to the force of the ball). This exponential expansion is called inflation.

\section{The monopole problem}

The exponential expansion during the inflationary epoch dilutes the relic particles like magnetic monopoles rapidly as the energy density falls slower than the relic particle density. So, the density of relic particles becomes negligible very quickly. However, for this scenario to work, the energy density at the end of the inflation should be enough to be turned into conventional matter without recreating the relic particles. For this, the temperature should not get high enough during the conversion process known as reheating. Only then can we get back to the Big Bang Theory to take the full benefit of all its fruits like nucleosysthesis and the cosmic microwave background (CMB) (Liddle, 1999).

When the table is considered to expand exponentially and the room's wall expands normally, the table's edges soon cross the room's wall i.e. the apples at the edge cross the wall. So, the magnetic monopoles reach beyond our particle horizon

\section{Conclusive words with hypothesis}

Chaotic inflation is a much better model of inflation as it doesn't require fine tuning unlike the new and old inflationary theories. The idea of eternal inflation seems reasonable and the fact that dark matter and dark energy can be used as some sort of indications of existence of 
parallel Universes-can be useful to support eternal inflation theory. Even though this hypothesis isn't verifiable, dark matter could be the effect of a parallel Universe which co-exists with ours. It could be the result of some higher dimensional Universe from where our Universe is visible but to whom we cannot see, just like an imaginary 2D TV character having height and width as the dimensions cannot see us in the dimension of depth. If that is the case, dark matter and dark energy could just be some higher dimensional features which have been unleashed to our Universe by the co-existing higher-dimension Universe. That being the case, the inflation field (which can actually resemble the Cosmological Constant or Dark Energy in some ways can be the result of the influence of higher dimension. A string-theory approach to test this hypothesis will be much appreciable in the future of Cosmology. However, a better approach is to deliver a mechanism that can be theoretically possible and experimentally verifiable.

If the early Universe had a rapid spin, the so-produced centrifugal force could rip apart many parts and throw them away. The space could have expanded in similar manner and inflation could indeed be not required. A sudden centrifugal force could have led to some sort of exponential expansion which was stopped very soon by the release of massive amount of energy in the process. If that is the case, Cosmic Inflation is more of a Cosmic Rotation and the inflation field is more of a Rotation Virtual Field. Even though this idea may seem unlikely, the extreme temperature in the early ages of the Universe could have triggered such mechanism. This is definitely testable and verifiable at least within couple of decades.

Fig.1 The figure shows evolution of scalar fields $\phi$ and $\varphi$ during self-reproduction process in the Universe (Linde, 2014)
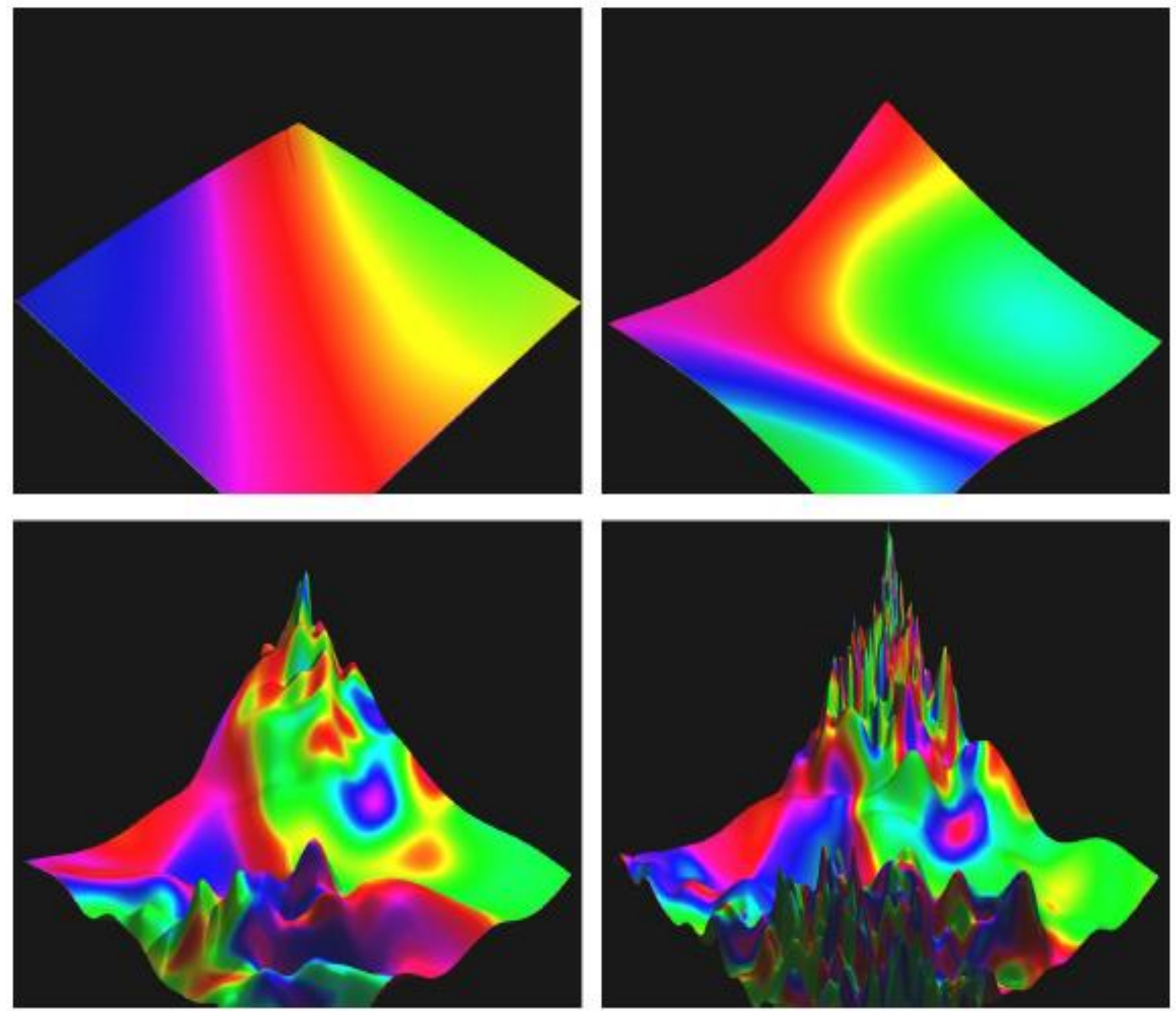
Fig.2 Solution of the horizon problem; The Hubble length is initially large which leads to formation of smooth patch by causal interactions; however, inflation later shrinks the Hubble length. Afterwards, the subsequent expansion of the Universe still leaves the observable Universe within smooth patch (Liddle, 1999)

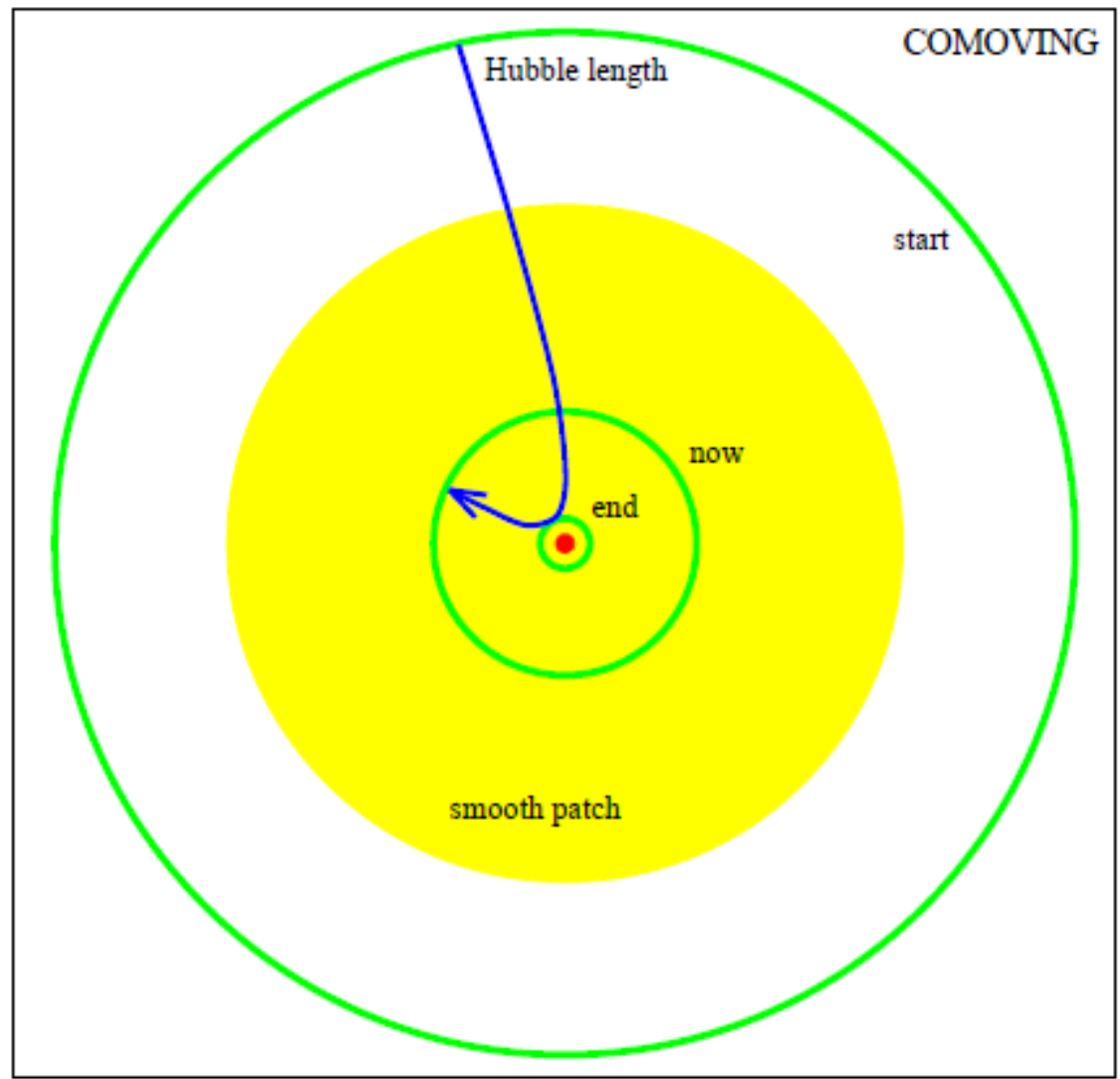

However, another aspect of analyzing the Universe would be to not expect everything to have a verifiable proof within our reach. We are so-called intelligent species whose intelligence is completely evaluated by comparison with the other un-intelligent life forms. Nonetheless, the possibility of the existence of much higher species is absolutely undeniable and we trying to explain the mysteries of Cosmic Inflation could be very similar to the monkeys trying to figure out the secrets of what appear on the night sky. We might be nothing more than a dog looking at the Moon and thinking it to be some sort of food that is beyond its reach. Just like the dog has no reference to relate to his observation of the Moon, we might have no reference to relate to the theory of creation of the Universe. In that scenario, it wouldn't be a surprise for us to not understand the mysteries of Cosmology because it isn't an astonishing fact as we know it that a dog has absolutely no idea what the stars might be. In a very similar manner, higher dimensional species might be evaluating us and laughing at the very idea of "humans trying to figure out the mysteries of
Cosmic Inflation" just like we laugh at the idea of "dog wondering how to get to the Moon."

Nevertheless, it is the responsibility of us scientists to try and figure out the mysteries of the Universe by means of different theories and observations. After all, what seemed absolutely inexplicable a century ago - like the source of fuel of the stars- are now a matter of fact known even to a $10^{\text {th }}$ grader - nuclear fusion fuels the stars. In a similar manner, what seems completely out of explanation to the greatest scientists of our time- like the mysteries of Dark Matter, Dark Energy and the source that triggered Cosmic Inflation- could very well be a well-known fact to a $10^{\text {th }}$ grader of the $22^{\text {nd }}$ Centuryprobably some sort of multiverse theory which is simple and verifiable from observations in our own Universe. It is thus the responsibility of us scientists to hand-over advanced scientific knowledge to our descendants, just like our ancestors handed over the knowledge to us. Building more powerful telescopes and even better theories could definitely be a great help for figuring out 
what these mysteries of the Universe are really explained by.

\section{References}

Preskill, (2004). J. Physical Review Letters. 43(19): 1365-1368.

Michio, K. Parallel Worlds.

A.R. (1996). LiddlearXiv:astro-ph/9612093v2 (1996).

A. Riotto arXiv:hep-ph/0210162 (2002).

Linde, A.D. (1974). JETP Lett. 19 (1974).

A.D. LindearXiv:0705.0164v2 (2008).

Starobinsky, A.A. (1979). JETP Lett. 30, 682.

Starobinsky, A.A. (1980). Phys. Lett. B 91, 99.

Mukhanov, V.F. and Chibisov, G.V. (1981). JETP Lett. 33,532 .
Guth, A.H. (1981). Phys. Rev. D 23, 347.

Linde, A.D. (1983). Phys. Lett. B 129, 177.

Gibbons, G.W. and Turok, N. (2008). Phys.Rev.D 77.

A D LindearXiv:1402.0526v2(2014).

A R Liddle arXiv:astro-ph/9901124 (1999).

Kofman, L., Linde, A.D., Starobinsky, A.A. (1994). Phys.Rev.Lett.73:3195-3198.

Bellini, M. (2002). Gen.Rel.Grav. 34.

Linde, A.D. (1994). Phys.Rev.D49:748-754.

A.D. Linde (1990).

A.D. Linde (1986). Phys.Lett. B 175, 395.

Goncharov, A.S., A.D. Linde, V.F. Mukhanov. (1987). Int. J. Mod. Phys. A 2, 561.

J. MakelaarXiv:1408.6435v1(2014).

A. H. Guth, (1981). Phys. Rev. D23, 347.

\section{How to cite this article:}

Nirakar Sapkota, Binod Adhikari. 2017. A Review on Cosmic Inflation. Int.J.Curr.Res.Aca.Rev. 5(5), 34-45. doi: https://doi.org/10.20546/ijcrar.2017.505.006 